\title{
PIDA Denetçilerin Bozucu Dışlama Performansının Teorik Incelenmesi
}

\author{
Necati Özbey ${ }^{1 *}$, Celaleddin Yeroğlu ${ }^{2}$, Barış Baykant Alagöz ${ }^{3}$ \\ ${ }^{1}$ İnönü Üniversitesi, Malatya MYO, Elektronik ve Otomasyon Bölümü, Malatya, Türkiye (ORCID: 0000-0002-2205-8890) \\ ${ }^{2}$ İnönü Üniversitesi, Mühendislik Fakültesi, Bilgisayar Mühendisliği Bölümü, Malatya, Türkiye (ORCID: 0000-0002-6106-2374) \\ ${ }^{3}$ İnönü Üniversitesi, Mühendislik Fakültesi, Bilgisayar Mühendisliği Bölümü, Malatya, Türkiye (ORCID: 0000-0001-5238-6433)
}

(İlk GelişTarihi 21 Ağustos 2019 ve Kabul Tarihi 22 Şubat 2020)

(DOI: 10.31590/ejosat.608644)

\begin{abstract}
ATIF/REFERENCE: Özbey, N., Yeroğlu, C. \& Alagöz, B. B. (2020). PIDA Denetçilerin Bozucu Dışlama Performansının Teorik İncelenmesi. Avrupa Bilim ve Teknoloji Dergisi, (18), 42-53.

$\ddot{O} \mathbf{z}$

Kontrol alanında ve endüstride çok yaygın kullanılan PID denetçiler yüksek dereceli sistemlerde bazen yetersiz kalmaktadırlar. Oransal Integral Türevsel Ivme (Proportional Integral Derivative Acceleration/PIDA) denetçilerin, Oransal Integral Türevsel (Proportional Integral Derivative/PID) denetçiye göre yüksek dereceli sistemlerde daha etkin cevap verdiği bazı güncel çalışmalarda gösterilmiştir. Ancak PIDA denetçilerde de çevresel bozucular ve sistem tarafindan oluşturulan iç gürültüler denetçi performansını olumsuz etkileyebilmektedir. Kapalı çevrim kontrol sistemlerinde referansa bozucu oranı (Reference to Disturbance Rate/RDR) ile gürültü bastırma kapasitesinin belirlenmesine, bozucu dışlama etkinliğinin artırılmasına katkı sağlayabilir. RDR indeksi kapalı çevrim kontrol sistemlerinin çıkışında referans giriş sinyalinin eklemsel giriş bozucu sinyale oranını ifade eder. Bu yayında önerilen bozucu bastırma kontrolü, çevresel bozucu etmenlerin denetçi performansı üzerindeki olumsuz etkilerinin azaltılmasını sağlayan bir denetçi tasarımını hedeflenmektedir. Çalışmada kapalı çevirim birim geri beslemeli bir sistemde Rastgele Arama (RandomSearch/RS) algoritması ile tasarlanan PIDA denetçinin bozucu dışlama performansı incelenmiştir.
\end{abstract}

Anahtar Kelimeler: PIDA denetçi, Bozucu dışlayıcı kontrol, Rasgele arama algoritması.

\section{Theoritical Investigation of Disturbance Rejection Performance of PIDA Controllers}

\begin{abstract}
Proportional Integral Derivative (PID) controllers, which are the most widely used in the field of control and industry, sometimes become insufficient in higher order systems. Proportional Integral Derivative Acceleration (PIDA) controllers were suggested to respond more effectively than PID in high-order systems in some up-to-date references. But, environmental disturbances and internal noise generated by the system can seriously affect PIDA controller performance. In negative unity feedback closed-loop control systems, investigating the Disturbance Rejection Capacity with the Reference to Disturbance Rate (RDR) may contribute disturbance rejection performance. RDR defines the ratio of the output signal and the noise signal of the system. The control of disturbance rejection, proposed in this paper, aims to design a controller that reduces the negative effects of noise on control performance. The paper determines the disturbance rejection performance of PIDA controller designed with Random Search (RS) algorithm in a closed loop feedback system.
\end{abstract}

Keywords: PIDA Controller, Disturbance rejection control, Random search algorithm.

\footnotetext{
*Sorumlu Yazar: İnönü Üniversitesi, Malatya MYO, Elektronik ve Otomasyon Bölümü, Malatya, Türkiye, ORCID: 0000-0002-2205-8890, necati.ozbey@inonu.edu.tr
} 


\section{Giriş}

Kontrol bilimindeki gelişmeler hemen hemen her alanda hayatımızı olumlu yönde etkilemektedir [1-2]. Günümüzde oldukça yaygın kullanılan PI, PD ve PID denetçiler yüksek dereceli sistemlerde bazen yetersiz kalmaktadırlar. Son yıllarda, yüksek dereceli sistemlerin kontrolü için önerilen yöntemlerden biri de 1996'da Jung ve Dorf tarafından sunulan PIDA denetçilerdir. Bu sistemlerde PIDA denetçilerin PID’ye göre daha etkin cevap verdiği birçok çalışmada ileri sürülmektedir [3, 4]. Bu denetçilerin kolay kullanımının yanında bazı zayıf yanları da hala bulunmaktadır. Örneğin, çevresel bozucu etkiler ve sistem tarafindan oluşturulan iç gürültüler denetçi performansını ciddi bir şekilde etkileyebilmektedir. Bu yayında önerilen bozucu dışlayıcı kontrol yapısı, gürültülerin denetçi performansı üzerindeki olumsuz etkilerini azaltan bir denetçi tasarımını hedeflemektedir. Günümüze kadar PID denetçilerin bozucu dışlama kapasitesi üzerine çeşitli çalışmalar yapılagelmiş ve birçok öneriler ileri sürülmüştür [5-15]. Kapalı çevrim kontrol sistemlerinde RDR ile bozucu dışlama kapasitesinin belirlenmesi, kontrol sistemlerinin bozucu dışlama performansı açısından önemlidir [5-7]. RDR indeksi bazı çalışmalarda denetçi tasarımında [8,16] ve bozucu dışlama performansının incelenmesinde [9] uygulanmıştır.

Pratik kontrol uygulamalarında, çevresel etkiler ve sistem tarafından oluşturulan iç gürültüler denetçi performansını ciddi bir şekilde etkileyebilmektedir. Bozucu dışlayıcı kontrol yaygın olarak iki yöntemle uygulanmaktadır;

i. Açık Yaklaşım Yöntemleri: Filtreler, gürültü ve durum gözleyiciler, gürültü tahmin ediciler, uyarlanabilir durum geri besleme denetçileri gibi ek ifadeler ve ek bloklar ekleyen yöntemler [17-20].

ii. Kapalı Yaklaşım Yöntemleri: Denetçilerin yapısal olarak RDR özelliklerini geliştirerek önceden tanımlı bir modele göre, kararlılık ve kutup yer değiştirmeye dayalı yöntemler [21, 22]. Öngörülemeyen bozucu etkilere karşı dayanıklı kontrol sistemleri için, kontrol sisteminin duyarlılık fonksiyonunun (sensitivity function) sınırlanması [10] ve RDR indeks performansının artırılmasına dayanan tasarım yöntemleri öneren yaklaşımlar [5-7].

$\mathrm{Bu}$ yayında kapalı çevirim geri beslemeli bir sistemde RS algoritması ile RDR indeksini maksimize ederken yerleşme noktası kontrol (set-point kontrol) performansını iyileştiren çoklu-amaç fonksiyonlu PIDA denetçisi tasarım yöntemi sunulmuştur. Temelde, yerleşme noktası kontrol performansı ile bozucu dişlayıcı kontrol performansı arasında ters ilişki vardır [7]. Tasarım probleminin ana hedefi, hem bozucu dışlama performansı hemde yerleşme noktası kontrol performansının kabul edilebilir derecede iyi olmasını sağlayan bir PIDA denetçi katsayılarının belirlenmesidir. Bu probleme çözüm için uzaşlama eğrisi tabanlı bir pareto optimal çözüm yöntemi olan RS algortiması adapte edilmiştir. Bu amaçla, PIDA denetçi için RDR indeks formülasyonu çıkarılmış, uzlaşma eğrisi tabanlı çok amaçlı RS algortiması PIDA denetçi tasarımı için düzenlenmiştir. Önerilen bu tasarım yöntemin sonuçları ve etkinliği iki PIDA denetçi tasarım örneği ile incelenmiştir. Tasarlanan PIDA kontrol sistemlerinin bozucu dışlama kapasitesi ve giriş bozucularına karşı dayanıklılığı incelenmiştir.

$\mathrm{Bu}$ makalenin organizasyonu şöyledir: Gelecek bölümde tasarım yönteminde uygulanan teorik altyapı sunulmuştur. Üçüncü bölümde PIDA denetçi için RDR indeksi formulasyonu elde edilmiş ve bu formülasyonun uzlaşma eğrisi tabanlı çok amaçlı RS algortimasına uygulanması açıklanmıştır. Dördüncü bölümde, düzenlenen RS algortiması ile iki PIDA denetçi tasarım örneği üzerinde sistemin bozucu dışlayıcı ve yerleşme noktası kontrol perfromansları incelenmiştir.

\section{Teorik Altyapı}

\subsection{Rasgele Arama Algoritması}

Sistemlerin istenen uygulama performanslarını ortaya koyabilmeleri için optimal olarak tasarıma gereksinim duyulur. Bu nedenle sistem tasarımında optimizasyon yöntemlerinin kullanılabilir. Sezgisel optimizasyon algoritmaları denetçi tasarımlarında yaygın olarak kullanım alanı bulurlar. Yapılacak tasarım işlemlerinin amacı ihtiyaç duyulan kontrol parametrelerinin değerlerini belirlemek ve en iyi değerleri bir optimizasyon algoritması ile bulup hedeflenen sonuca ulaşmaktır [23]. Model tabanlı kontrol sistemi tasarımında kontrol edilen sistemi temsil eden matematiksel model ve denetçi fonksiyonundan oluşan kontrol sistemi üzerinde optimizasyon algortimaları yardımı ile istenen kontrol performansını sergileyen optimal denetçi katsayıları belirlenir. Sistem karmaşıklığ 1 ve performans kriterleri arttıkça optimal denetçi tasarım problemi zorlaşır ve bu noktada sezgisel yöntemler kolaylık sağlar. Bunun temel nedeni sezgisel yöntemler ile uygun aday çözümlerin oluşturulması ve sistemde denenerek, her iterasyonda aday çözümlerin iyileştirilmesi prensibidir. $\mathrm{Bu}$ süreç temelde birbirini takip eden deneme yanılma işlemlerine dayanır.

RS algortiması parametre uzayında rastgele arama için önerilen temel bir algoritmadır ve yerel minimum değerini rasgele arama sonucunda bulan düşük maliyetli bir yöntemdir [24]. Bu algortimanın düşük işlem maliyeti donanımsal gerçeklemelerde sistem performasının ve hızının artmasına imkân sağlar. Dolayısı ile gerçek zamanlı uygulamalar için diğer karmaşık metasezgisel yöntemlere göre etkin ve kolay kullanım imkânı sağlar. Ayrıca, RS algortiması, sürü tabanlı yöntemlere göre deneme-yanılma işlemini daha tasarruflu kullanılır ve bu açıdan gerçek zamanlı pratik uygulama için daha avantajlıdır. Ayrıca, kontrol sistemlerinin zaman bölgesi optimizasyonlarında sürü tabanlı yöntemler sıklıkla kararsız çözümlere yol açarak optimizasyon sürecini zorlaştırmaktadır. Fakat aday sürüsü yerine tek bir çözümü rastgele adımlarla arayan RS algortiması kontrol sisteminin zaman bölgesi tasarım optimizasyonlarında daha düşük olasılıkla kararsız çözümleri denemesi nedeni ile çok daha uygundur. Bu yöntemin temel adımları şöyle özetlenebilir.

Adım 1: Rastgele bir başlangıç aday çözüm oluştur.

Adım2: Aday çözümü optimizasyon probleminde denenir ve uygunluk değeri alınır. 
Adım3: Uygunluk değeri yeterince iyi veya maksimum iterasyon sayısına gelinmişse optimizasyonu durdurulur. Diğer durumlarda Adım 4'e gidilir.

Adım 4: Aday çözümü rastgele değiştirilir ve Adım 2'ye dönülür.

Üçüncü bölümde RS algortimasının bozucu dışlayıcı PIDA denetçi tasarım probleminde uygulanması için uzlaşma eğrisi tabanlı çok amaçlı optimizasyon için geliştirilmesi gösterilmiştir.

\subsection{PIDA Denetçi Yapısı}

PIDA denetçiler klasik PID denetleyiciye ivme ilave edilerek elde edilir ve yüksek dereceli sistemlerde daha etkin cevap verdiği ileri sürülmektedir. Jung ve Dorf tarafından 1996'da önerilen [3], PIDA denetçinin geçiş fonksiyonu şöyle verilmektedir.

$C(s)=K_{p}+\frac{K_{i}}{s}+K_{d} s+K_{a} s^{2}$

Bu denetçi tasarımı için önerilen Dorf Yaklaşımı [25], Katsayılı Diyagram Metodu [26], Kitti's Metodu [27] literatürde iyi bilinen yöntemler arasındadır. Ayrıca literatürde PIDA tasarımında bazı optimizasyon algoritmalarını kullanan çalışmalar da mevcuttur. Örneğin, optimum PIDA denetçi, harmoni arama algoritması kullanılarak grafiksel olarak ve performans endeksleri ITAE, IAE ve ISE açısından değerlendirilmiştir [28]. PIDA denetçi tasarımı üzerine yapılan çalışmalardan bir diğeri de denetleyici parametrelerinin, çeşitli optimizasyon algoritmaları, kullanılarak elde edilmesi ve bazı performans endeksleri açısından değerlendirilmesidir [29-31]. PIDA denetçi ile kontrol edilen birim geri beslemeli bir sistem Şekil 1'de gösterilmiştir.

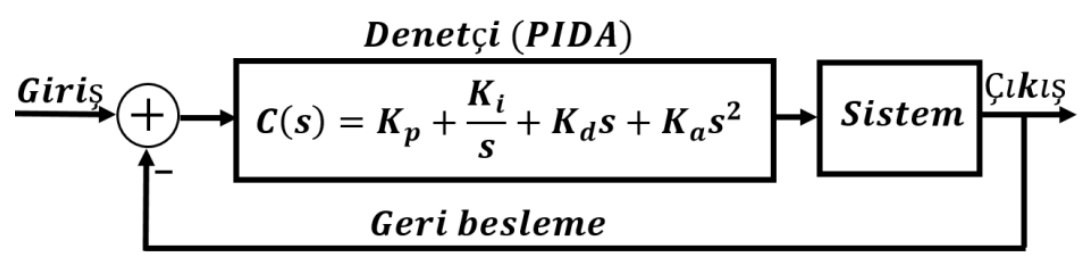

Şekil 1. Kapall çevrim PIDA kontrol sistemi

Bu çalışmada Şekil 1'de temsil edilen kapalı çevrim PIDA kontrol sisteminin bozucu dışlama performansı ve yerleşme noktası performansının optimal olarak kontrolünü sağlamak ve gürültü bastırma oranını belirlemek için RS algoritması geliştirilmiştir. Gerçek kontrol uygulamalarında bozucu etkilere karşı dayanıklı uygun denetçi parametrelerinin belirlenmesi kritik öneme sahiptir [13, 32]. Gerçek dünyada kontrol sistemleri genellikle tahmin edilemeyen çevresel etkilere maruz kalmaktadır. Çeşitli denetçi yapılarının kontrol sisteminde bozucu etkilerini bastırdığı pek çok çalışma yapılmıştır. Bu nedenle, gerçek dünyadaki kontrol uygulamalarında sistemin bozucu dışlama performansı dikkate alınmalıdır. Kontrol mühendisliği uygulamalarında kapalı çevrim kontrol sistemi tasarımında bozucu dışlama performansı ve yerleşme noktası kontrol performansı arasında ters ilişki olduğu bilinmektedir. Bunun başlıca nedeni şöyle açıklanabilir; Kapalı çevrim kontrol sistemlerinin eksemsel giriş bozucu dışlama performansı, kullanılan denetçinin daha güçlü olması ile sağlanır. Güçlü denetçi, yüksek kazançlı denetçi tasarımı ile mümkün olabilir. Bununla birlikte, yüksek kazançlı denetçiler referans girişin keskin değişikliklerinde çok yüksek aşımlara ve yerleşme noktasına kadar salınımlara neden olur. Bu durum yüksek aşım ve salınımlı yerleşme, yerleşme noktası kontrol performasını düşürür. Hem güçlü denetçi ile bozucu etkilerin bastırılması hem de yerleşme noktası kontrol performasının iyileştirilebilmesi için alçak geçiren karakterde giriş filtresi referans işarete uygulanır. Böylece, keskin değişimlere yol açan yüksek frekanslı bileşenler referans giriş işaretinden süzülerek kontrol sisteminde yüksek aşım ve yerleşme öncesi salınımlar önlenmeye çalışılır [7]. Bu tasarım yerleşme noktası filtreli iki-serbestlik dereceli kapalı çevrim kontrol olarak adlandırılır [7,33]. Tasarlanacak PIDA denetçinin yüksek kazanç katsayıları ile sistemin bozucu dışlama performansı artırılabilir.

\subsection{RDR Analizi ve Bozucu Bastırma}

Kapalı çevrim kontrol sistemlerinde RDR ölçütü, giriş bozucusu dışlama kapasitesini açısal frekansa bağlı olarak ifade etmektedir ve kapalı çevrim kontrol sisteminin bozucu dışlama performansının iyileştirilmesini sağlamak için optimizasyon problemlerinde kıstas veya amaç fonksiyonu olarak uygulanmıştır $[8,16]$. Bu çalışmada, PIDA denetçi ile kontrol edilen kapalı çevrim kontrol sisteminin bozucu dışlama kapasitesi ölçütü kullanılarak incelenmiştir. RDR analizi, sistem çıkışındaki referans sinyal enerjisinin sistem gürültü sinyalinin enerjisine oranın ile tanımlanır. RDR $>>1$ olması kontrol sisteminin gürültü bastırma performansının iyi olduğunu, RDR $<<1$ olması kontrol sisteminin gürültü bastırma performansının yetersiz olduğunu gösterir[5-7]. Bir önceki bölümde RDR ölçütü ile kontrol hatasının (yerleşme noktası performansı) ters ilişkili olduğu açıklanmıştı. İyi bir denetçi tasarımda optimizasyon işlemi RDR ölçütünün yüksek olmasını ancak kontrol hatasının (e) düşük olmasını sağlamalıdır. Bu nedenle, RS algortimasında RDR ölçütü ile kontrol hatası arasında uzlaşmayı sağlayan bir eğri yardımı ile pareto optimal optimizasyon sağlanmıştır. Optimizasyon işlemi bu eğriye göre yönetilerek eğri üzerinde optimal bir performans nokatasına ulaşılmaya çalışılmaktadır. 
Geri beslemeli kapalı çevrim kontrol sistemleri için bu güne kadar çok çeşitli gürültü bastırma ve bozucu dışlama yöntemleri önerilmiştir [18,32]. Ogata, bir kontrol sistemi çıkışında gürültünün bastırılması için gerekli koşulları denetçi, sistem ve kapalı çevrim transfer fonksiyonları bakımından ifade etmiş̧ir. Birim geri beslemeli bir kapalı çevrim kontrol sisteminin gürültü bastırma performansını iyileştirmek için $\left|C_{(s)} G_{(s)}\right| \gg 1$ durumunun olması gerektiğini önermiştir [34]. Çünkü yüksek açık çevrim kazancı durumunda $\left(\left|C_{(s)} G_{(s)}\right| \gg 1\right)$, gürültü girişli bir sistemin transfer fonsiyonu olan $P_{d}(s)=Q(s) / d(s)$ sıfir değerine yaklaşabilmektedir. Bozucu dışlama performansı kapalı çevrim sistemin duyarlılık fonksiyonunun minimize edilmesi ile iyileştirilmektedir [10]. Daha sonra, kontrol uygulamaları için kapalı çevrim kontrol sisteminin bozucu dışlama kapasitesi RDR ölçütü ile karakterize edilmiştir [5-7].

Şekil 2'de verilen girişine filtre $(F(s)=0$ ) eklenmiş bir lineer sistem için $d(s)=0$ durumunda referans girişinden sistem çıkışına referans işaret kanalı geçiş fonksiyonu $P_{r}(s)$ ve $r(s)=0$ olması durumunda bozucu girişinden sistem çıkışına bozucu işaret kanalı geçiş fonksiyonları $P_{d}(s)$ şöyle yazılabilir [6,7]:

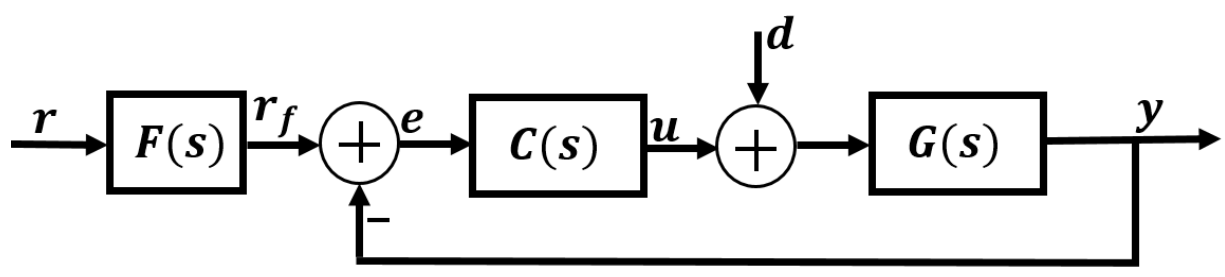

Şekil 2. Filtre eklenmiş PIDA kontrol sistemi.

$P_{r}(s)=\frac{\left.Q_{r}(s)\right|_{d=0}}{r(s)}=\frac{C(s) G(s)}{1+C(s) G(S)}$

$P_{d}(s)=\frac{\left.Q_{d}(s)\right|_{r=0}}{d(s)}=\frac{G(s)}{1+C(s) G(S)}$

Bu geçiş fonksiyonları yardımı ile kapalı çevrim kontrol sisteminin çıkışındaki referans işaretin ve bozucu işaretin spektral gücü ise $s=j w$ dönüşümü uygulanarak şöyle ifade edilmiş̧ir [6,7]:

$\left|Q_{r}(j w)\right|_{d=0}^{2}=\left|P_{r}(j w) \cdot r(j w)\right|^{2}=\left|P_{r}(j w)\right|^{2} \cdot|r(j w)|^{2}$

$\left|Q_{d}(j w)\right|_{r=0}^{2}=\left|P_{d}(j w) \cdot d(j w)\right|^{2}=\left|P_{d}(j w)\right|^{2} \cdot|d(j w)|^{2}$

Haberleşme kanallarının sinyal iletim kalitesini ifade eden Sinyal Gürültü Oranı (SNR) ölçütüne benzer şekilde, kapalı çevrim sistemlerin bozucu dışlama kapasitesi RDR ölçütü

$R D R=\frac{\left|Q_{r}(j w)\right|^{2}}{\left|Q_{d}(j w)\right|^{2}}=\frac{\left|P_{r}(j w)\right|^{2} \cdot|r(j w)|^{2}}{\left|P_{d}(j w)\right|^{2} \cdot|d(j w)|^{2}}$

ile ifade edilmiştir. Burada bozucu dışlama performansının doğru ölçülebilmesi için referans işaretin enerjisi $|r(j w)|^{2}$ ile bozucu işaretin enerjilerinin eşit olduğu $|d(j w)|^{2}$ varsayılmıştır. Bu durumda RDR ölçütü,

$R D R=\frac{\left|P_{r}(j w)\right|^{2}}{\left|P_{d}(j w)\right|^{2}}=|C(j w)|^{2}$

İfadesi elde edilir. Burada, çok yüksek rakamsal değerleri nedeni ile RDR indeksinin desibel türünden ifade edilmesi tavsiye edilir.

$$
R D R_{d B}(w)=20 \log |C(j w)|
$$

Açısal frekansa $w$ bağlı ifade edilen RDR ölçütü her frekans bileşeni için kontrol sisteminin bozucu işareti referans giriş işaretine göre ne oranda dışlayacağını ifade eder. Pratik kontrol sistemleri genelde düşük frekans bölgesinde çalışan sistemlerdir. Bu nedenle kontrol sisteminin düşük frekans bölgesinde yüksek RDR değerlerine sahip olması bozucuya dışlama kapasitesinin yüksek olmasını sağlar. Bu nedenle bozucu dışlayıcı optimal denetçi tasarımı aşağıdaki kıstasa uyulması ile sağlanır $[8,16]$.

$\min \left\{R D R_{d B}(w)\right\} \geq M, w \in\left[w_{\min }, w_{\max }\right]$

Bu kıstas $w \in\left[w_{\text {min }}, w_{\text {max }}\right]$ bölgesinde RDR performasının bir alt limit olan $M \in R$ değerinden yüksek olmasını garanti eder. 


\section{3. ÖnerilenTasarımYöntemi}

\subsection{PIDA Denetçi için RDR Ölçütünün Elde Edilmesi}

Denklem 1'de verilen PIDA denetçinin frekans cevabı transfer fonksiyonunda $s=j w$ kullanılarak,

$$
C(j w)=K_{p}+\frac{K_{i}}{j w}+K_{d} j w+K_{a}(j w)^{2}
$$

elde edilebilir. Gerekli düzenlemeler yapılırsa PIDA denetçinin genliği şöyle yazılır,

$$
|C(j w)|=\sqrt{\left(k_{p}-k_{a} w^{2}\right)^{2}+\left(k_{d} w-\frac{k_{i}}{w}\right)^{2}}
$$

Buna göre PIDA denetçisi için RDR ölçütü denklem (7)'ye göre,

$$
R D R_{P I D A}(w)=\left(k_{p}-k_{a} w^{2}\right)^{2}+\left(k_{d} w-\frac{k_{i}}{w}\right)^{2}
$$

ile ifade edilir. Burada $k_{p}, k_{d}, k_{i}$ ve $k_{a}$ PIDA denetçisi kazanç katsayılarıdır. Bir PIDA denetçinin tasarımında istenilen kontrol cevabını elde etmek için bu 4 tasarım parametresi optimal olarak belirlenir. Şekil 3'de tasarım katsayıların 1 olması durumu için $\left(k_{p}=1, k_{d}=\right.$ $1, k_{i}=1$ ve $k_{a}=1$ ) PIDA ve PID denetçi RDR performansları, $0<w<1000 \mathrm{rad} / \mathrm{s}$ bölgesinde incelenmiştir. Şekilde PIDA denetçi $1 \mathrm{rad} / \mathrm{s}$ frekansı civarında PID denetçiden daha düşük RDR performansı göstermesine rağmen düşük, orta ve yüksek frekans bölgelerinde genelde daha iyi RDR performansı sergilediği görülmektedir. Ancak, $1 \mathrm{rad} / \mathrm{s}$ frekansa sahip bir bozucunun gelmesi durumunda sistemin çıkışında güçlenerek RDR değerini -30 dB değerlerine düşürdüğü șekilde görülmektedir.

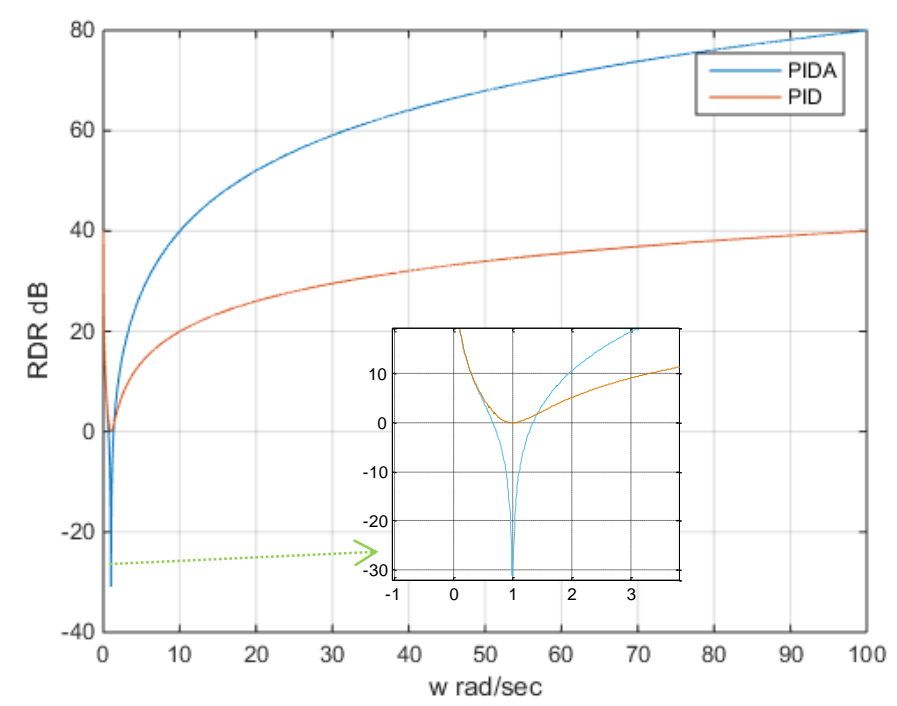

Şekil 3. Kapalı çevrim PIDA ve klasik PID kontrol sistemnini RDR spektrumları

\subsection{Uzlaşma Eğrisi Tabanıı RS Algortiması Yardımı ile Bozucu Dışlayıcı PIDA Denetçi Tasarımı}

Bu bölümde PIDA kontrol sisteminin, bozucu dışlama performansı dikkate alınarak, optimal tasarımı için uzlaşma eğrisi tabanlı RS algoritması uygulanmıştır. Şekil 2'de görüldüğü gibi birim geri beslemeli PIDA denetçi sistemine yerleşme noktası filtresi $F(s)$ eklenmiştir. Ön filtre $F(s)$, girişdeki çok yüksek frekans bileşenlerini filtrelemek üzere referans giriş sinyalini şekillendirmek için kullanılan birinci dereceden bir filtredir [21]. Bunun nedeni, RDR endeksleri yüksek olan denetçilerin referans girişindeki hızlı değişikliklere karşı çok güçlü ve duyarlı olmalarıdır. Denetçinin bu hassasiyeti birim basamak performansının azalmasına neden olur. Çünkü buradaki yüksek aşımlar yüksek frekans bileşenleri içerir. Bu yüksek aşımları azaltmak için, ön filtre, $F(s)$, birim basamak girişini düzeltir. Böylece birim basamak tepkisi bozulmadan kontrol sisteminin RDR'1, daha da artırılabilir ve hem bozucu dışlama hemde yerleşme noktası kontrol performanslarının iyileştirilmesi sağlanır. Bu sistemde bozucu sinyal $(d)$, kontrol edilen sisteme dışardan gelen ve kontrol sinyalinin üstüne ilave eklenen dış kaynaklı bir gürültü işareti olarak varsayılmıştır.

Şekil 2'de kullanılan ön filtre fonksiyonu şöyle yazılır,

$F(s)=\frac{a}{s+a}$

Burada $a=1 / \tau$ ve $\tau$ zaman sabitidir. Kontrol sisteminin birim basamak performansı, karesel hata toplamı ile şöyle değerlendirilir. 
$E=\frac{1}{T} \int_{0}^{T} e(t)^{2} \cdot d t$

$T$ 'nin bir periyot içinde $e(t)=r_{f}(t)-y(t)$ kontrol hatasının genliğinin ( $e$ 'nin) en aza indirilmesi ve sistem çıkışının $y(t)$, referans girişe $r(t)$ yaklaştırılması kontrol sisteminin temel hedefidir. Öte yandan, kontrol sisteminin bozucu dışlama performansını artırmak için Denklem 9'da verilen minimum RDR şartının Denklem 12 ile ifade edilen PIDA denetçisi RDR ölçütü için sağlanması gerekmektedir. Önerilen bu kontrol tasarım yönteminin birincil amacı, sistemi kararlı tutmak ve ayar noktasını takip etmektir. İkincil amacı ise denetçinin minimum RDR seviyesinin birincil amacın sağlandı ̆̆ı koşulda artırılmasıdır. Aksi takdirde kontrol uygulaması için düşük kontrol edilebilirliğe sahip yüksek gürültü bastırma istenmez. Bu durum, çok amaçlı optimizasyon problemini gündeme getirir. Bu optimizasyon problemi minimum RDR kısıtlamalarının dinamik formu olan aşağıda verilen kısıt için çözülmüştür.

$\min \left\{10 \log \left(R D R_{P I D A}(\mathrm{w})\right\} \geq M(E)\right.$

$M(E)=-\propto \log _{10} E, w \in\left[w_{\min }, w_{\max }\right]$

Burada minimum RDR kısıtlaması için $M(E)$ dinamik alt sınırdır. $E$ 'nin azalması bir $\propto$ faktörü tarafından minimum alt sınırın $M(E)$ artmasına neden olur. Denklem 16 uzlaşma eğrisi olarak adlandırılır. Burada $\propto$ parametresi kabul edilebilir bir minimum RDR ve istenilen bir $E$ özelliğini karşılamak için kullanılan logaritmik bir konsensüs katsayısıdır ve aşağıdaki formda belirlenebilir,

$\propto=\frac{\min \left(10 \log \left(R D R_{P I D A}(\mathrm{w})\right)\right.}{\log _{10} E}$

Şekil 4'de uzlaşma eğrisinin $M(E)$ 'nin $\propto$ parametresinin 4 değeri için bir çizimi görülmektedir. Bu eğri optimizasyonda kabul edilebilir minimum RDR için bir dinamik alt sınır ifade eder ve kontrol karesel hata toplamı $E$ ile RDR ölçütü arasında bir uzlaşma sağlar. Optimizasyon işlemine uzlaşma eğrisi üzerinde veya üstünde kalan taralı bölgede izin verilir.

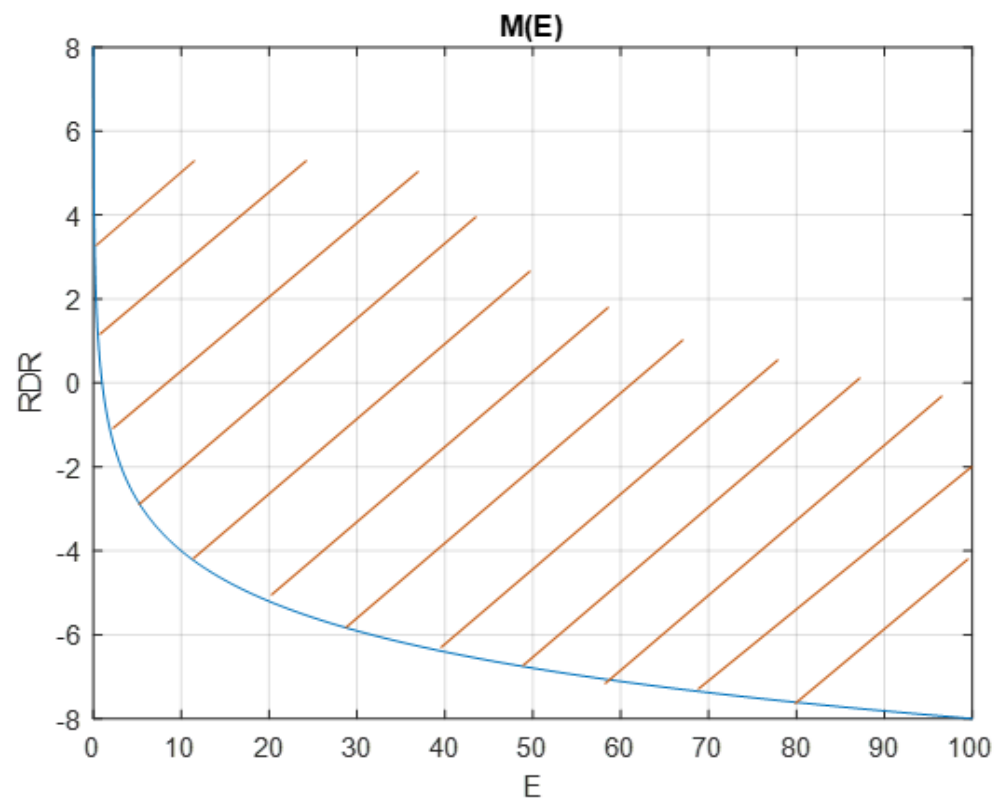

Şekil 4. RDR ile karesel hata toplamı (E) ilişskisi.

Bu optimizasyon problemini çözmek için önerilen temel RS algoritmasının işlem basamakları şöyle özetlenebilir;

- $\quad$ Adım 1: $k_{p}, k_{d}, k_{i}$ ve $k_{a}$ denetçi parametrelerinin başlangıç değerleri, sistemi karalı yapan herhangi bir değere ayarlanır. Filtre parametrelerinin başlangıç değerleri $a$ ve $E_{\min }$ için yüksek bir değer belirlenir.

- Adım 2: Aşağıdaki verilen öz yinelemeli eşitliklerle arama uzayında rasgele yeni aday noktaları oluşturulur,

$k_{p n}=k_{p}+($ rand -0.5$) c_{g}$;

$k_{d n}=k_{d}+($ rand -0.5$) c_{g}$;

$k_{\text {in }}=k_{i}+($ rand -0.5$) c_{g} ;$ 
$k_{a n}=k_{a}+($ rand -0.5$) c_{g} ;$

$a_{n}=a+($ rand -0.5$) c_{f}$

- $\quad$ Adım 3: Çalışma frekans aralığını belirlemek için $w \in\left[w_{\min }, w_{\max }\right], \min \left\{R D R_{d B}\right\}$ ve aralığı ile birim basamak tepkisi için $E$ hata fonksiyonu hesaplanır.

- Adım 4: $E_{\min }$ minimum hata için $M\left(E_{\min }\right)=-\propto \log _{10} E_{\min }$ dinamik RDR sınırı hesaplanır.

- Adım 5: Eğer $E<E_{\text {min }}$ ve $\min \left\{R D R_{d B}\right\} \geq M\left(E_{\min }\right)$ ise $k_{p}=k_{p n}, k_{i}=k_{i n}, k_{a}=k_{a n}, a=a_{n}$ değerlerini ve minimum enerji $E_{\min }=E$ güncelleştirilir.

- Adım 6: $E_{\min }$ yeterince küçükse veya maksimum yenileme sayısı aşılırsa, optimizasyon sonlanır. Aksi takdirde 2. adıma gidilir.

Burada $c_{g}$-kazanç katsayıları ve $c_{f}$-filtre katsayıları ilgili parametrelerin rasgele değişim aralığını belirler.

\section{Simülasyon Örnekleri}

PIDA tasarım problemlerinde RDR performansının bir tasarım kriteri olarak kullanılması, kapalı çevrim kontrol sistemlerinin gürültü bastırma kapasitelerinin artııılmasına katkı sağlaması öngörülmüştür. RDR tasarım sınırlamasının kullanılması arama bölgelerini daraltması nedeni ile sezgisel optimizasyon algoritmalarının etkinliğini artırır. Bu bölüm teorik bilgileri doğrulamak için gürültü toleranslı PIDA denetçi örneklerini göstermektedir. Bu örneklerde Matlab Simulink kullanılmıştır. Optimizasyonda parametrelerin rastgele değişim aralığı $c_{g}=0.05$ ve $c_{f}=0.2$ olarak alınmıştır.

\section{Örnek 1 :}

Bu örnekte $G(s)=1 /\left(s^{2}+4 s+3\right)$ olarak verilen kararlı ikinci dereceden bir sistemin transfer fonksiyonu için PIDA denetçi tasarımının yerleşme noktası ve bozucu dışlama performansı incelenmiştir. Bu sistem için tasarım adımları uygulanarak aşağıdaki PIDA denetçi elde edilmiş ve RDR performansı incelenmiş̧ir.

$C_{P_{P D A_{-} 1}(s)}=3.3058+\frac{5.0320}{s}+1.3897 s+0.0174 s^{2}$

Şekli 5'de PIDA denetçi tasarımı için RDR spektrumu görülmektedir. Özellikle sıfır ve çok düşük frekans bölgesinde çok yüksek RDR performansı ortaya koymaktadır ve bu frekans bölgesinde referans işaretin kontrol sistemi çıkışında çok daha baskın olacağını ifade etmektedir. Açısal frekansı $1.8 \mathrm{rad} / \mathrm{s}$ civarında olan frekans bileşenleri için RDR performansının minimum olduğu görülmektedir ve bu frekansda çevresel bozuculara karşı bozucu dışlama performansı en kötü olacaktır. Yüksek frekans bölgesinde RDR indeksi tekrar yükselerek özellikle yüksek frekans bölgesinde etkili olan beyaz gürültüye karşı kontrol sisteminin dayanıklı olacağına işaret etmektedir. Şekil 6 PIDA denetçinin optimizasyonu esnasında tasarım parametrelerinin değişimini vermektedir. Şekil 7 ise amaç fonksiyonu olan kontrol hatasının optimizasyon süresince değişimini göstermektedir. Bu grafikte hata değerinin sıfira yakınsaması ve asimptotik olması optizasyonun gerçekleştiğini ve kontrol performansının minimum RDR alt sınır değeri ile uzlaşarak optimize edildiğini göstermektedir. Optimizasyon tamamlandığında $\min \left\{R D R_{d B}\right\}=10.2192 d B$ için $E_{\min } 0.0060$ değerine kadar kontrol hatasının düşmesi sağlanmıştır.

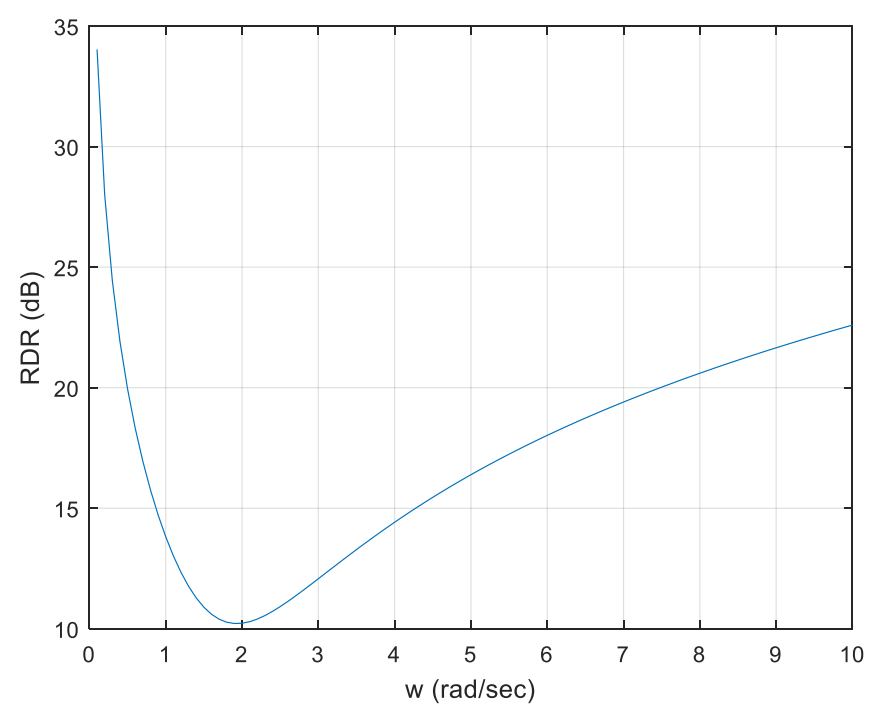

Şekil 5. PIDA denetçinin gürültü bastırma spektrumu. 


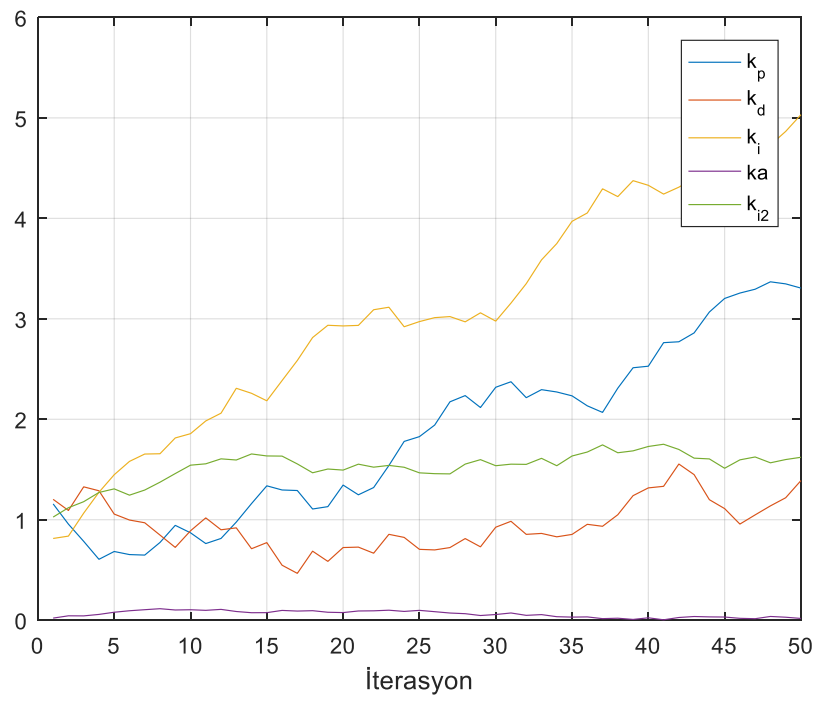

Şekil 6. Optimizasyon işlemi sırasında tasarım parametresinin değişimi.

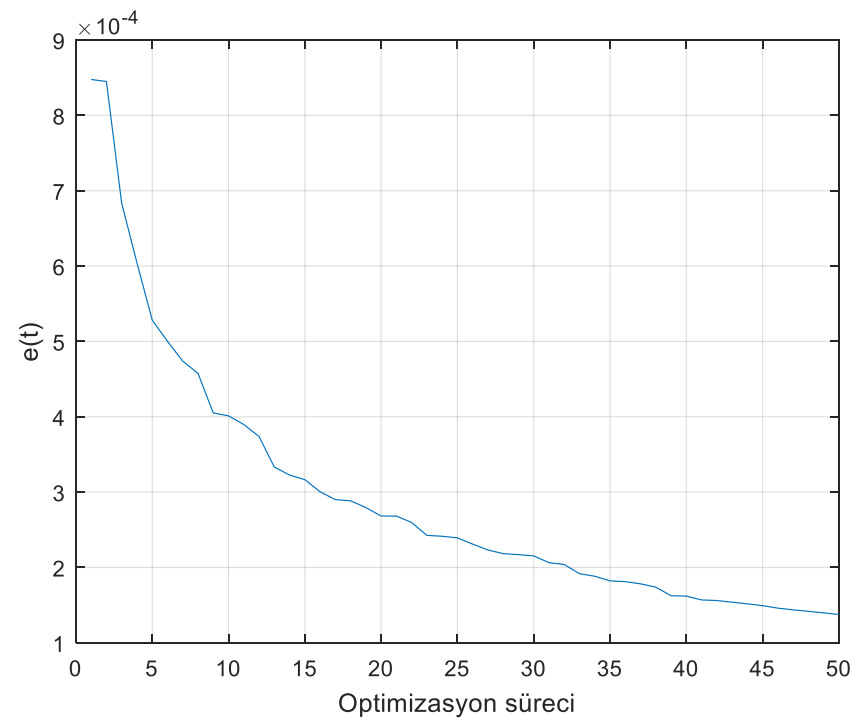

Şekil 7. Optimizasyon sırasında E'nindeğişimi

Şekil 8'de Denklem 19'daki PIDA kontrol sistemi ve klasik PID kontrol sisteminin $(k p=5.3211 ; k i=6.1205 ; k d=0.8199)$ birim basamak cevabı ve bozucu cevapları simülasyon sonucu olarak sunulmuştur.

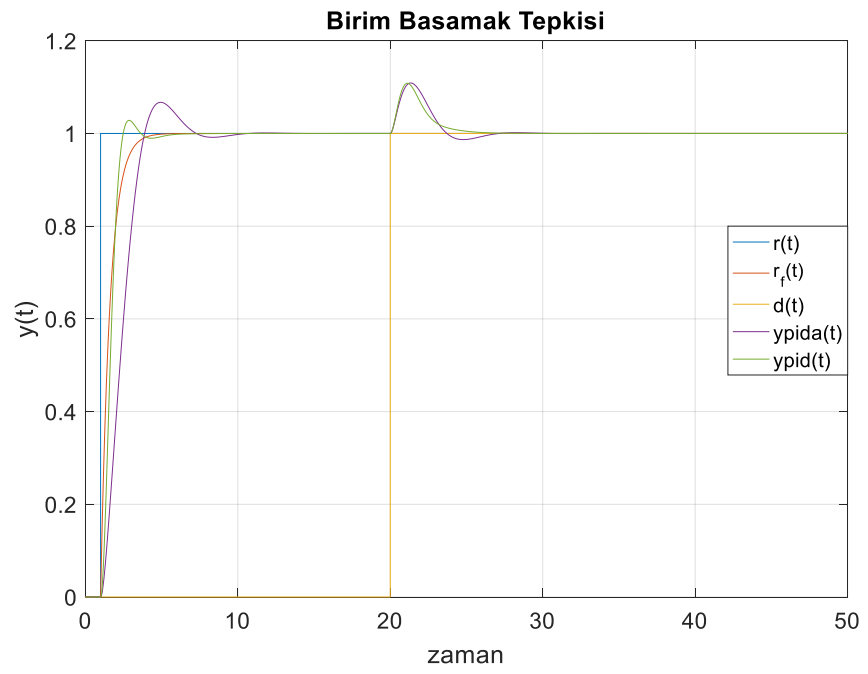

Şekil 8. Optimize edilmiş PIDA ve PID denetçilikontrol sisteminin birim basamak tepkisi 
Burada 20. saniyede birim basamak formunda bozucu sinyal kontrol edilen sistem girişine uygulanmış ve her iki kontrol sistemi için birim basamak tepkisi elde edilmiştir. Şekillerde PIDA denetçinin klasik PID'ye göre daha iyi kontrol performansı sunabildiği açıkça görülmektedir. Bu sonuçlar yerleşme noktası filtreli iki-serbestlik dereceli kapalı çevrim PIDA denetçi tasarımının hem yerleşme noktası kontrol performansını hemde bozucu dışlama performansını birlikte iyileştirebildiğini göstermiştir. Bu örnekte uygulanan uzlaşma eğrisi tabanlı RS algortimasının iyi performans sergilediği söylenebilir.

\section{Örnek 2:}

Bu örnekte yüksek dereceli $G(s)=1 /\left(s^{4}+4 s^{3}+6^{2}+4 s+1\right)$ olarak verilen bir sistemin transfer fonksiyonu için PIDA denetçi tasarımının yerleşme noktası ve bozucu dışlama performansı incelenmiştir. Uygulanan yöntemle elde edilen PIDA denetçi aşağıda verilmişsir.

$C_{P I D A_{\_} 2}(s)=34.2243+\frac{26.9797}{s}+10.5161 s+1.0779 s^{2}$

Şekli 9'da PIDA denetçi tasarımı için RDR spektrumu görülmektedir. Sıfır ve çok düşük frekans bölgesi RDR performansı oldukça yüksektir. Bu çıkışta referansın sıfır ve düşük frekans değişimlerinde baskın olacağını diğer bir ifade ile çıkışın referans girişe yakınsayacağını gösterir. En kötü RDR performansı $2 \mathrm{rad} / \mathrm{s}$ frekans civarında elde edilir. Çünkü burada RDR spektrumu minimum değerine sahiptir ve bu bölgede bozucu işaretin sistem çıkışındaki gücünün artması beklenir. Yüksek frekans bölgesinde RDR indeksinin yükselmesi özellikle yüksek frekans bölgesinde etkili olan beyaz gürültü gibi bozuculara karşı kontrol sisteminin dayanıklı olacağına işaret etmektedir. Şekil 10'daki optimizasyon süresince amaç foksiyonun değişimi görülmektedir. Burada $\min \left\{R D R_{d B}\right\}=29.7847 d B$ için $E_{\min } 0.0002$ değerine kadar inmektedir. Şekil 11'de Denklem 20'deki PIDA ve klasik PID denetçilerin $(k p=2.0542 ; k i=$ $0.5001 ; k d=2.0928$ ) birim basamak ve bozucu cevapları gösterilmiştir. Bu şekilde 20. saniyede bir birim basamak bozucu sinyal uygulanmış ve tasarlanan PIDA ve klasik PID denetçilerinin bu bozucuya cevapları incelenmiştir. Bu örnekte PIDA'nın hem yerleşme noktası kontrol performansı hemde bozucu dışlama performansının PID denetçiye göre çok daha üstün olabileceği görülmüştür. Bu örnekte ugulanan optimizasyon yönteminin hem birim basamak yerleşme noktası cevabını hem de bozucu dışlama performansını iyileştirebildiği ve klasik PID denetçiye kıyasla avantajlar sağlayabildiği görülmüştür.

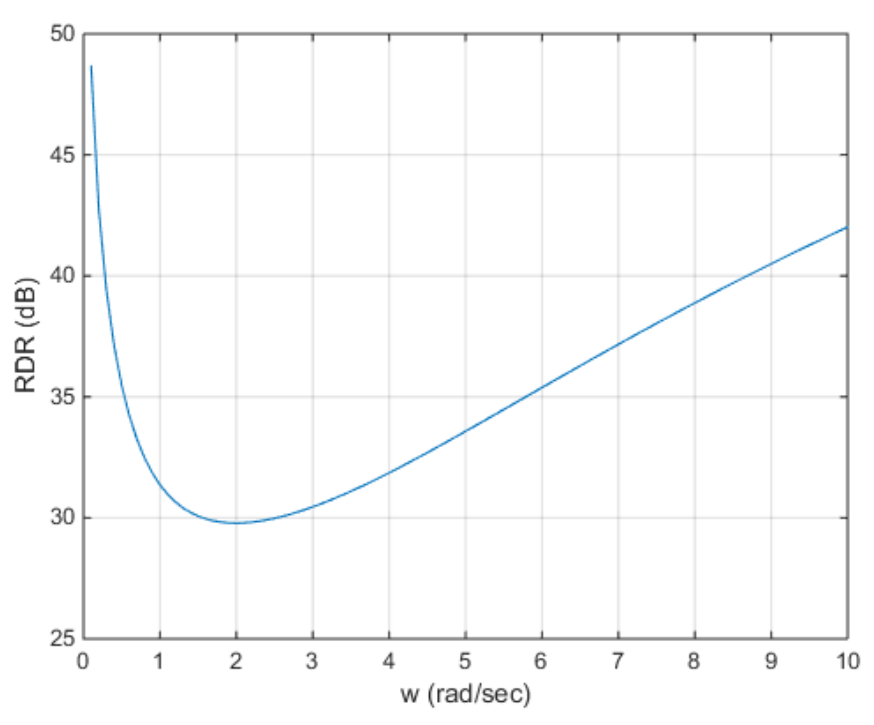

Şekil 9. PIDA denetçinin gürültü bastırma spektrumu. 


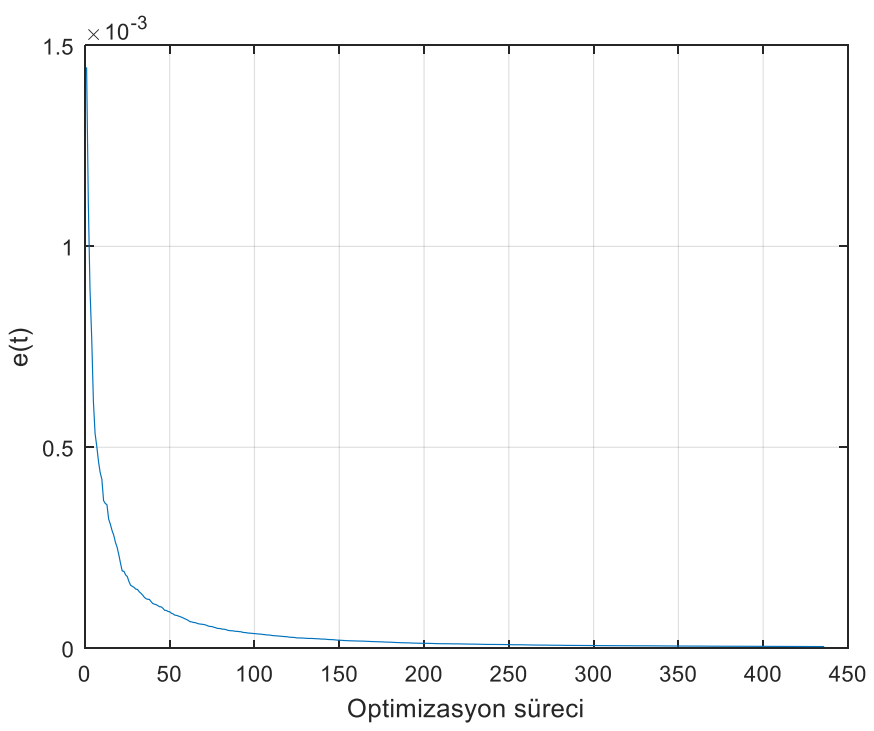

Şekil 10. Optimizasyon sırasında E'nin değişimi.

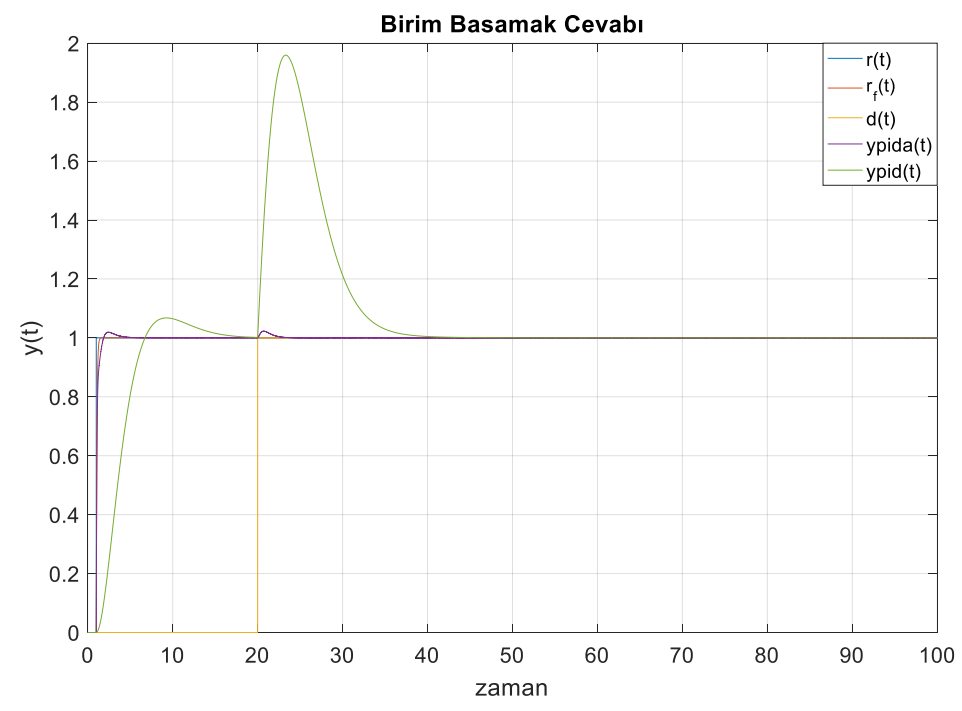

Şekil 11. Optimize edilmiş PIDA vePID denetçilikontrol sisteminin birim basamak tepkisi.

\section{Sonuç}

$\mathrm{Bu}$ çalışmada, yerleşme noktası filtreli iki-serbestlik dereceli kapalı çevrim PIDA denetçi tasarımı için RDR performansı analizine dayalı çok amaçlı RS algortimasının bir uygulaması gerçekleştirilmiştir. Bu uygulamada tasarlanan PIDA denetçilerin performansları klasik PID denetçilere kıyasla incelenmiş ve kontrol simülasyonları sonucunda tasarlanan PIDA denetçilerin birim basamak yerleşme noktası kontrol performansı ile bozucu dışlama performanslarının birlikte iyileştirilebildiği gösterilmiştir. Bu sonuca ulaşabilmek için PIDA denetçisinin RDR indeksi matematiksel olarak elde edilmiş ve uzlaşma eğrisi tabanlı bir Pareto optimizasyonu yaklaşımı uygulanmıştır. Böylece, temel RS algoritmasının optimizasyonu önceden tanımlanan uzlaşma eğrisine göre yönlendirilmiş ve RDR performansının yeterice yüksek olduğu bölgede kontrol hatasının minimizasyonu sağlanmıştır. Makalede literatürdeki temel çalışmaların üzerinde PIDA denetçi için hem RDR indeks formülasyonu hemde uzlaşma eğrisi tabanlı RS algortimasının uygulaması geliştirilmiştir. Bu yöntem ile tasarlanan denetçiler Matlab/Simulink ortamında geliştirilen simülasyonlarda test edilmiş ve bozucu dışlama performanları incelenmiştir.Bu çalışmada, birim geri beslemeli kontrol sistemlerinde denetçi fonksiyonunun enerji spektral yoğunluğu ile ifade edilen RDR performansının kontrol sistemlerinin bozucu dışlama performansının iyileştirilebilmesinde etkin bir şekilde uygulanabileceği görülmüştür. Kontrol sistemin RDR analizi, denetçilerin gürültü bastırma kapasitesinin nitel olarak değerlendirmesini sağlamanın yanında denetçi ayarlama sorunları için yararlı ve basit bir analitik çözüm sunduğu gösterilmiştir. Diğer taraftan, bu çalışmada PIDA denetçilerin bozucu dışlama kontrolü performansları RDR spektrumları ile detaylı olarak incelenmektedir. Elde edilen sonuçlar kontrol tasarım uygulamalarında önerilen PIDA denetçi yapısının ve uygulanan optimizasyon yönteminin sistemlerin dayanıklı kontrol performansının artırılmasına katkı sağlayacağı öngörülmüştür. 


\section{Kaynakça}

[1] Kuo B. C., Golnaraghif.,(2010). Automatic Control Systems,9th Ed., Wiley Press, USA.

[2] Dorf, R. C. Bishop, R. H., (2010). Modern Control Systems, 12th Ed., Prentice Hall, New Jersey, USA.

[3] Jung, S. Dorf, R. C., (1996).Analytic PIDA Controller Design Techniquefor A Third OrderSystem.Proceedings of the 35th Conference on Decisionand Control, Kobe, Japan, pp. 2513-2518. DOI: 10.1109/CDC.1996.573472.

[4] Ha, D-Y. Lee, I-Y. Cho, Y.S. LimY-D. Choi, B-K. (2001).The Design of PIDA Controller withPre-compensator.Proceedings of the IEEE International Symposium ISIE , Pusan, Korea, pp. 798-804, DOI:10.1109/ISIE.2001.931570.

[5] Deniz, F. N., Keles, C., Alagoz, B. B., \& Tan, N. (2014, June). Design of fractional-order PI controllers for disturbance rejection using RDR measure. In ICFDA'14 International Conference on Fractional Differentiation and Its Applications 2014 (pp. 1-6). IEEE.

[6] Alagoz, B. B. Deniz, F. N. Keles, C. Tan, N. (2015). Disturbance Rejection Performance Analyses of Closed Loop Control Systems by Reference to Disturbance Ratio. ISA Transactions, vol. 55, pp. 63-71, DOI: 10.1016/j.isatra.2014.09.013.

[7] Alagoz, B. B., Tan, N., Deniz, F. N., Keles, C. (2015). Implicit disturbance rejection performance analysis of closed loop control systems according to communication channel limitations. IET Control Theory \& Applications, vol. 9(17), pp. $2522-2531$.

[8] Ates, A., Alagoz, B. B., Yeroglu, C., Yuan, J., \& Chen, Y. (2017, August). Disturbance rejection FOPID control of rotor by multiobjective BB-BC optimization algorithm. In ASME 2017 International Design Engineering Technical Conferences and Computers and Information in Engineering Conference (pp. V009T07A025-V009T07A025). American Society of Mechanical Engineers.

[9] Tepljakov, A., Alagoz, B. B., Gonzalez, E., Petlenkov, E., \&Yeroglu, C. (2018). Model reference adaptive control scheme for retuning method-based fractional-order PID control with disturbance rejection applied to closed-loop control of a magnetic levitation system. Journal of Circuits, Systems and Computers, 27(11), 1850176.

[10]Monje, C. A., Vinagre, B. M., Feliu, V., \& Chen, Y. (2008). Tuning and auto-tuning of fractional order controllers for industry applications. Control engineering practice, 16(7), 798-812.

[11]Vrancic, D. Strmcnik, S. Kocijan, Moura Oliveira, J. P. B. (2010). Improving Disturbance Rejection of PID Controllers by Means of the Magnitude Optimum Method. ISATransactions, vol. 49, pp. 47-56,DOI:10.1016/j.isatra.2009.08.002.

[12]Krohling, A. R. Rey, J. P. (2001). Design of Optimal Disturbance Rejection PID Controllers Using Genetic Algorithms IEEE Transactions on Evolutionary Computation, Vol. 5, No. 1, pp. 78-82, DOI:10.1109/4235.910467.

[13]Ahmad, A. A. Hussein, E. M. (2014). Effect of Disturbance on Closed-Loop Control System. IJIREST, Vol.3, Issue 8, pp.1567215676, DOI:10.15680/IJIRSET.2014.0308080

[14]Chen, D. Seborg, D. E. (2002). PI/PID Controller Design Based on Direct Synthesis and Disturbance Rejection. Ind. Eng. Chem. Res., Vol. 41, pp.4807-4822, https://doi.org/10.1021/ie010756m.

[15]Vandeursen, J. E. Peperstraete, J. A. (1996). Model-basedand PID Controllers for Disturbance Rejection in Processes with Time Delay: AComparison. ISATransaction 35, pp. 225-236, https://doi.org/10.1016/S0019-0578(96)00031-6.

[16]Özbey, N. Yeroğlu, C. Alagöz, B.B. (2018). A Set-point Filter Type 2DOF Fractional Order PID Control System Design Scheme for Improved Disturbance Rejection Control. The International Conference on Fractional Differentiation and its Applications (ICFDA) ,16-18 July 2018, Amman. DOI:10.2139/ssrn.3273677.

[17]Alsogkier, I. Bohu, C. (2017). Rejection and Compensation of Periodic Disturbance in Control Systems. IJEIT, Vol.4, No.1, pp.4454.

[18]Chang, J. L. (2011). Robust Output Feedback Disturbance Rejection Control by Simultaneously Estimating State and Disturbance. Journal of Control Science and Engineering, pp. 1-13, http://dx.doi.org/10.1155/2011/568379.

[19]Busawon, K. K. Kabore, P. (2001). Disturbance Attenuation Using Proportional Integral Observers. International Journal of Control 74:6, pp. 618-627, https://doi.org/10.1080/00207170010025249.

[20]Shamsuzzoha, M. Lee, M. (2009). Enhanced Disturbance Rejection for Open-loop Unstable Process with Time Delay. ISA Transactions, Vol.48, Issue 2, pp. 237-244, https://doi.org/10.1016/j.isatra.2008.10.010.

[21]Szita, G. Sanathanan, C. K. (1997). "Robust Design for Disturbance Rejection in Time Delay Systems. J. Franklin Inst., Vol. 334B, No.4, pp. 611-629, https://doi.org/10.1016/S0016-0032(96)00090-7.

[22]Koussiouris, T. G. Tzierakis, K.G. (1996). Frequency-domain Conditions for Disturbance Rejection and Decoupling with Stability or Pole Placement Automatica, Vol.32, No.2, pp. 229-234, https://doi.org/10.1016/0005-1098(96)85552-X.

[23]Price, W. L. (1983). Global Optimization by Controlled Random Search. Journal Of Optimization Theory and Applications, Vol. 40, No. 3, pp. 333-348, https://doi.org/10.1007/BF00933504.

[24]Andradóttir, S. (1999). Accelerating the Convergence of Random Search Methods for Discrete Stochastic Optimization. Journal of Association for Computing Machinery, Vol. 9, No. 4, pp. 349-380, DOI:10.1145/352222.352225.

[25]Sambariya, D. K. Paliwal, D. (2016). Comparative Design and Analysis of PIDA Controller Using Kitti's and Jung-Dorf Approach for Third Order Practical Systems. British Journal of Mathematics \& Computer Science Vol. 16, No. 5, pp.1-16, DOI : 10.9734/BJMCS/2016/26223

[26]Photong, P. Kampanaya, D. Komine, N. Ngamwiwit, J. (2000). Application of CDM to PIDA control. ASCC 3rd, July 3-7, Shanghai, TD-9-4, pp. 2073-2078.

[27]Ukakimaparn, P. Pannil, P. Boonchuay, P. Trisuwannawat, T. (2009). PIDA Controller Designed by Kitti's Method. ICROS-SICE, International Joint Conference, August 18-21, https://ieexplore.ieee.org/abstract/document/5335323.

[28]Sambariya, D. K. Paliwal, D. (2016). Optimal Design of PIDA Controller Using Harmony Search Algorithm for AVR Power System. IEEE 6th International Conference on Power Systems (ICPS),pp. 1-6. 4-6 March, DOI:10.1109/ICPES.2016.7584219.

[29] Sambariya, D .K. Paliwal, D. (2016). Optimal Design of PIDA Controller Using Firefly Algorithm for AVR Power System. International Conference on Computing, Communication and Automation (ICCCA), pp. 987-992, DOI:10.1109/ CCAA.2016.7813859. 
[30]Donuk, K. Özbey, N. İnan, M. Yeroğlu, C. Hanbay, D. (2018). PIDA Denetçi Parametrelerinin PSO Algoritması ile Belirlenmesi. 2018 International Conference on ArtificialIntelligenceand Data Processing (IDAP), 28-30 Sept. 2018 , pp. 107-112. DOI: 10.1109/IDAP.2018.8620871

[31] Karadeniz, E. Özbey, N. Yeroğlu, C. Kahraman, H.T. (2018). SOS Algoritması ile Tasarlanan PIDA Denetçinin Bozucu Bastırma Etkisi. 2018 International Conference on Artificial Intelligence and Data Processing (IDAP), 28-30 Sept. 2018, pp.549-553. DOI: 10.1109/IDAP.2018.8620800.

[32]Rojas, A. J. (2009). Signal-to-noise Ratio Performance Limitations for Input Disturbance Rejection in Output Feedback Control. Systems \& Control Letters, Vol.58:5, pp. 353-358, https://doi.org/10.1016/j.sysconle.2009.01.001.

[33]Ateş, A., \& Yeroğlu, C. (2016). Online tuning of two degrees of freedom fractional order control loops. Balkan Journal of Electrical and Computer Engineering, 4(1), 5-11.

[34]Ogata, K. (2010). Modern Control Engineering, 5th Ed., Pearson Education, New Jersey, USA. 